\title{
Diagnostic and Monitoring Serology in Commercial Poultry Integrations: Practical Applications ${ }^{1}$
}

\author{
Gary D. Butcher, DVM²
}

Serologic testing is an important tool used in the commercial poultry industry for diagnosis of disease and monitoring of flock health. However, the proper methods for serologic testing are often not understood. It is essential to know the uses and limitations of this procedure to ensure maximum benefit is realized.

A distinction must be made between serologic testing for diagnostics and serologic testing for flock monitoring. Diagnostic serology attempts to relate a disease condition to rising antibody titers. Serology for flock monitoring is performed to evaluate a poultry health program. Diagnostic serology and monitoring serology are similar in that the results of both are expressed as antibody titers and the titers are determined by identical laboratory techniques. When samples are submitted for serologic testing, the testing should be performed in the same laboratory, by the same test system, and by the same laboratory technician. Samples for testing should be randomly collected and representative of the flock.

When a chicken is vaccinated or exposed to a disease, it responds by producing antibodies against that specific disease organism. Remember that a live vaccine is simply a mild form of the disease. The level of antibodies present in the blood are measured and expressed as titer values.

Antibody levels in the blood are constantly changing (i.e., increasing or decreasing). Conclusions can be made for diagnostic purposes only if we know which direction the titers are moving. The direction the antibody titers are going is determined by comparing two sets of serum samples taken over a 3to 4-week time span. The first sample is taken at the onset of the disease and the second approximately 4 weeks later. If titers are rising, this indicates recent exposure to the disease. If titers are decreasing, no recent exposure has occurred.

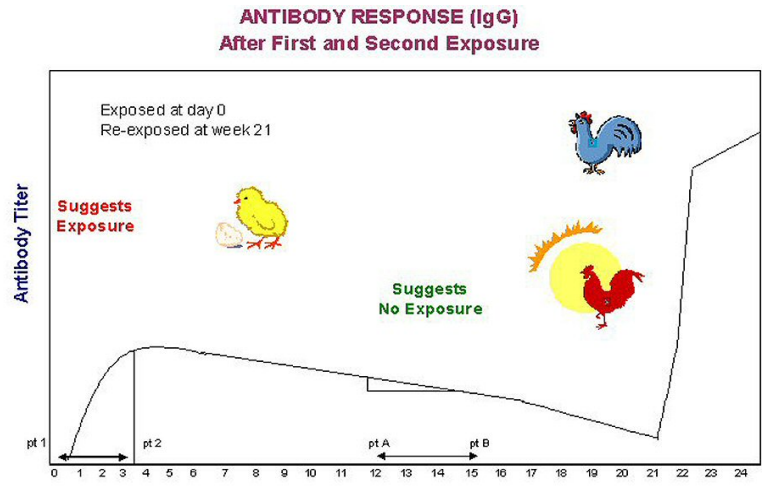

Figure 1.

1. This document is VM137, one of a series of the Veterinary Medicine-Large Animal Clinical Sciences Department, Florida Cooperative Extension Service, Institute of Food and Agricultural Sciences, University of Florida. Original publication date July 2, 2002. Visit the EDIS Web Site at http://edis.ifas.ufl.edu.

2. Gary D. Butcher, DVM, Ph.D., Diplomate, American College of Poultry Veterinarians, University of Florida College of Veterinary Medicine, Gainesville, FL.

The Institute of Food and Agricultural Sciences is an equal opportunity/affirmative action employer authorized to provide research, educational information and other services only to individuals and institutions that function without regard to race, color, sex, age, handicap, or national origin. For information on obtaining other extension publications, contact your county Cooperative Extension Service office. Florida Cooperative Extension Service/Institute of Food and Agricultural Sciences/University of Florida/Christine Taylor Waddill, Dean. 
Flock monitoring allows for evaluation of the health program and indicates when changes are needed based upon fact. Flock monitoring can help you determine:

1. Effectiveness of the pullet vaccination program

2. Need for boosting of breeders/layers during the production cycle

3. Maternal antibody titer levels

4. Plan for vaccinating chicks

5. Efficacy of vaccine administration

6. Exposure to a disease to which a vaccine has not been administered

When developing a monitoring program, remember that collecting too many serum samples can result in confusion, while too few samples may not provide enough information. An organized monitoring schedule should be developed so valid interpretations of the poultry health program can be made. Sample collection for monitoring pruposes will depend on the specific objectives for the testing.

Limitations of ELISA serology:

1. Measures IgG response only (blood level), not $\operatorname{IgA}, \operatorname{IgM}, \mathrm{CMI}$ or non-specific immune mechanisms

2. Must have paired sera to make a determination for diagnostic serology.

3. Must have an organized sample collection schedule for monitoring purposes

4. Antigenic specificity will effect testing results

5. Selection of samples is critical, need samples randomly selected and a sufficient nimber collected

6. Selection of birds is critical, ensure they represent the flock on disease condition

7. Understand that there is commonly some lack of consistancy of results among labs 